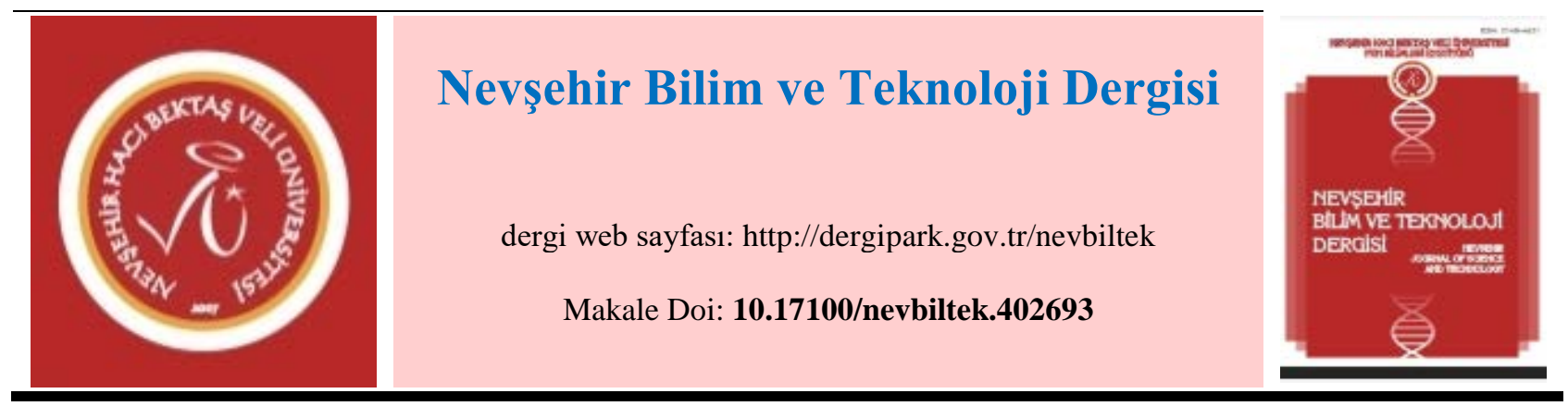

\title{
3 Fazlı Evirici Devresi için SDGM, SHEDGM, HIDGM ve UVDGM Tekniklerinin
}

\section{Karşılaştırılması}

\author{
Ayşe Kocalmış Bilhan' ${ }^{1}$, Sedat Sünter ${ }^{2}$ \\ ${ }^{1}$ Nevşehir H.B.V. Üniversitesi, Mühendislik Mimarlık Fakültesi, Elektrik Elektronik Mühendisliği Bölümü, Nevşehir \\ ORCID ID:0000-0002-5008-6784 \\ ${ }^{2}$ Firat Üniversitesi, Mühendislik Fakültesi, Elektrik Elektronik Mühendisliği Bölümü, Elâzı̆̆
}

Öz

Alternatif Akım iletim sistemleri, motor sürücü sistemleri, gerilim kompanzatörü, kesintisiz güç kaynakları (UPS) gibi birçok alanda kullanılan eviricilerin temel görevi sabit bir Doğru Akım/Gerilim kaynağından genliği ve frekansı kontrol edilebilir bir Alternatif Akım/Gerilim kaynağı üretmektir. Eviriciler, tek fazlı ve üç fazlı olarak üretilebildikleri gibi, besleme kaynaklarına göre Gerilim veya Akım Kaynaklı Eviriciler olarak da sınıflandırılabilmektedirler. Bu çalışmada, günümüzde Evirici devrelerinde gerilim ve frekans kontrolünün gerçekleştirilmesi amacıyla kullanılan Sinüsodial Darbe Genişlik Modülasyon (DGM) tekniği, Seçili Harmonik Eliminasyon DGM tekniği, Harmonik İlaveli DGM tekniği ve Uzay Vektör DGM tekniğinin Matlab/Simulink benzetim modeli gerçekleştirilerek R-L yükü üzerinde akım ve gerilim dalga şekilleri karşılaştırılmalı olarak incelenmektedir.

Anahtar Kelimeler: Evirici, DGM, SDGM, HIDGM, SHEDGM, SVDGM.

\section{The Comparison of SPWM, SHEPWM, HIPWM and SVPWM Techniques on 3-Phase Inverter Circuit}

\begin{abstract}
The main usage reason of inverters which are used in many medium and high voltage applications such as motor driving systems, UPSs, voltage compensators and etc. converts Direct Current to Alternative Current. Inverters can be produced as single-phase and three-phase, or they can be classified as Voltage Source Inverters or Current Source Inverters according to their supply sources. In this study, 3-phase Inverter circuit which are used Sinusoidal Pulse Width Modulation Technique (SPWM), Selective Harmonic Elimination Pulse Width Modulation Technique (SHEPWM), Third Harmonic Injection Pulse Width Modulation Technique (THIPWM) and Space Vector Pulse Width Modulation Technique (SVPWM) feeding R-L load are presented by using MATLAB/Simulink program and current and voltage waveforms are given comparably.
\end{abstract}

Keywords: Inverter, PWM, SPWM, THIPWM, SHEPWM, SVPWM

\footnotetext{
1 e-mail: akbilhan@gmail.com
} 


\section{Giriş}

Günümüzde elektrik enerji tüketiminin hızla artması ve sınırlı fosil enerji kaynaklarının olması, araştırmacıları alternatif enerji kaynaklarının geliştirilmesine ve var olan enerjinin daha fazla nasıl verimli kullanılabileceği konularında araştırmaya sevk etmektedir. Bu amaçla güneş pilleri, aküler, rüzgar türbinleri, yakıt pilleri gibi alternatif enerji gün geçtikçe daha yaygın olarak kullanılmaya başlanmıştır. Ancak gerek alternatif kaynaklar kullanılarak üretilen gerek ise doğrultucular kullanılarak elde edilen Doğru Akımın (DA) endüstride ve diğer birçok alanda Alternatif Akıma (AA) dönüştürülmesi gerekmektedir. Bu amaçla evirici adı verilen güç elektroniği devreleri kullanılmaktadır. Eviriciler en genel hali ile doğru gerilim üretilen bir kaynaktan, arzu edilen genlik ve frekansa sahip, simetrik bir alternatif gerilim üretmek amacıyla kullanılan güç elektroniği dönüştürücüleridir. 1980’li yıllarda güç elektroniği anahtarlarındaki gelişim (özelikle GTO, IGBT, GCTs’nin geliştirilmesi) yüksek güç ve orta gerilim gerektiren uygulamalarda (asenkron motor sürücülerinde, çimento endüstrisinde fanlarda, su pompalama istasyonlarında, nakliye endüstrisinde çekiş uygulamalarında, metal endüstrisinde vb.) eviricileri daha popüler hale getirdi. Günümüzde pompalar, taşıyıcı bant sisleri, fanlar, kompresörler vb. uygulamaların yaklaşık \%85 inde eviricilerin bulunduğu sürücü sistemler kullanılmaktadır [14]. Bu durum tüm sistemin çalışması değerlendirildiğinde enerji kaybını oldukça azaltmaktadır.

Eviricilerden bu dönüştürme sırasında beklenen en önemli özellik çıkışlarına istenilen gerilim ve çalışma frekansına ulaşılması ve elde edilen gerilim/akım bozulmalarının düşük seviyede olmasıdır. Evirici giriş gerilimi güneş paneli, akü, rüzgar türbinleri gibi kaynaklardan elde edilebileceği gibi doğrultucu devresinden de elde edilebilir.

Eviriciler tek fazlı ve üç fazlı olarak kullanılabildikleri gibi, besleme kaynaklarına göre; akım kaynaklı evirici ve gerilim kaynaklı evirici olarak iki çeşidi bulunmaktadır. Şekil 1 ile üç fazlı evirici devresi gösterilmiştir. Özellikle beslenen yükün, harmonik akımlara karşı yüksek empedans gösterme durumu varsa gerilim kaynaklı eviriciler kullanılmaktadır. Bu nedenle, endüstride gerilim kaynaklı eviricilerin büyük bir kullanım alanı mevcuttur.

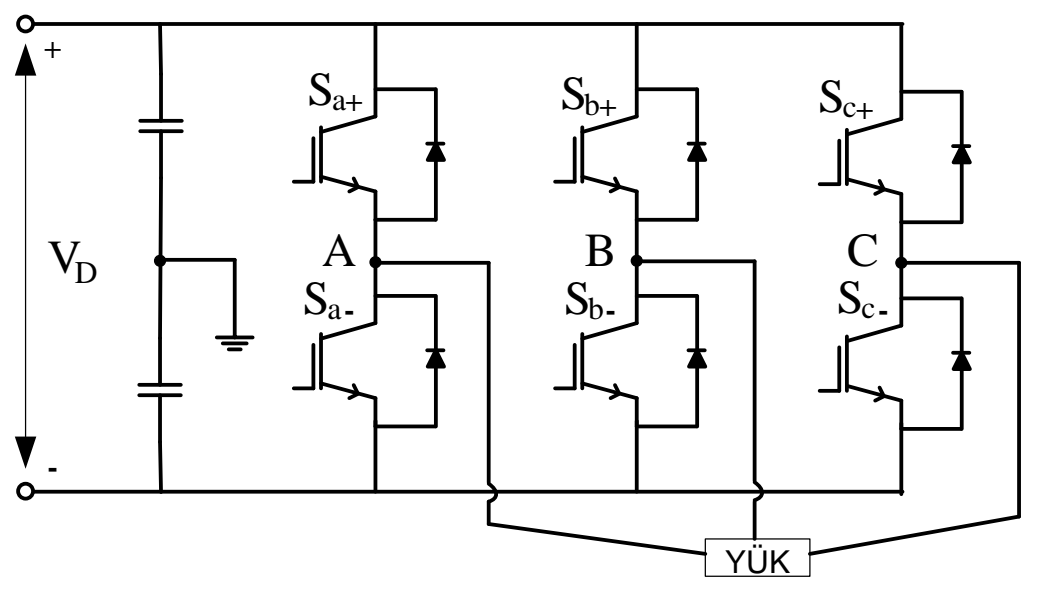

Şekil 1. Gerilim kaynaklı üç fazlı evirici devresi

Her faz için iki adet IGBT anahtarın $\left(\mathrm{S}_{\mathrm{a}^{+}}, \mathrm{S}_{\mathrm{a}-}, \mathrm{S}_{\mathrm{b}^{+}}, \mathrm{S}_{\mathrm{b}-}, \mathrm{S}_{\mathrm{c}^{+}}, \mathrm{S}_{\mathrm{c}-}\right)$ kullanıldığı Şekil 1 ile gösterilen evirici devresinde çıkış geriliminin genliğinin ve frekansının ayarlanabilmesi için darbe genişlik modülasyon (DGM, Pulse Width Modulation - PWM) adı verilen çeşitli teknikler geliştirilmiştir [5]. Geliştirilen her bir DGM tekniği ile anahtarlama kayıplarının azaltılması, lineer modülasyon aralığının genişletilmesi ve özellikle evirici çıkışında elde edilen gerilim ve akım dalga şekillerinde bulunan harmoniklerin azaltılması ve temel harmonik bileşenin genlik frekansının denetimi amaçlanmaktadır [6]. Gerek asenkron gerek senkron makinelerin çalışması sırasında oluşan harmonikler; demir ve bakır kayıplarının artmasına sebep oldukları gibi şebeke üzerinde rezonans oluşmasına da sebep olurlar.

Anahtarlama kayıplarının önem arz etmediği evirici devrelerinde yüksek frekansta çalışan DGM teknikleri (Sinüzoidal DGM (SDGM), Harmonik İlaveli DGM (HİDGM), yada Uzay Vektör DGM (UVDGM)) kullanılarak düşük harmonik bozulmalı (THD) arzu edilen genliğe sahip gerilim üretilir [5]. Anahtarlama kayıplarının önemli etkilerinin olduğu sistemlerde ise düşük frekansta çalışan DGM tekniklerinin (Seçili Harmonik Eliminasyon DGM-SHEDGM) kullanılması daha avantalı olmaktadır [3,7]. 


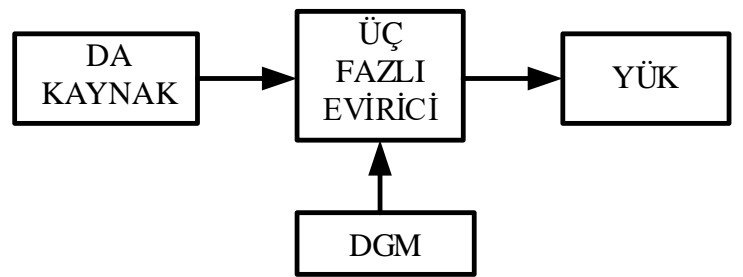

Şekil 2. Üç fazlı eviriciye ait blok diyagramı

Bu çalışmada sırasıyla SDGM, SHEDGM, HIDGM ve UVDGM yöntemlerinin üç fazlı tam köprü evirici devresine uygulanması durumunda elde edilen akım/gerilim dalga şekilleri incelenmektedir. Şekil 2'de üç fazlı evirici için kullanılan DGM teknikleri ait blok diyagram gösterilmektedir.

\section{Tam Köprü Evirici Devresinde SDGM Yöntemi}

SDGM tekniği evirici anahtarlarının iletim kesim durumlarının belirlenmesinde kullanılan en eski yöntemlerden biridir. Bu teknikte, $V_{\text {T }}$ genlikli ve $f_{\text {T }}$ frekanslı taşıyıcı bir sinyal (üçgen dalga) ile genliği $V_{s}$ ve frekansı fs olan bir modülasyon sinyali (sinüs dalga) karşılaştırılarak evirici devresindeki anahtarlar için DGM işaretleri üretilir. Üç fazlı evirici devrelerinde ise, Taşıyıcı dalga, dengeli üç fazlı cıkış gerilimleri elde etmek icin Şekil 3 (a) ile gösterildiği gibi üç tane aralarında $120^{\circ}$ faz farklı bulunan sinuzoidal kontrol gerilimleri ile karşılaştırılır. Şekil 3(b)'de ise karşılaştırma sonucunda her faz için uygulanacak sinyaller gösterilmektedir.

SDGM'de; modülasyon dalgasının genliğinin taşıyıcı dalganın genliğine oranına $\mathrm{M}_{\mathrm{I}}$ (modülasyon indeksi) olarak ifade edilmektedir ve bu oran 0 ile 1 arasında değişen bir değer arasındaysa modülasyon ve çıkış dalgası arasındaki ilişki lineer olur. SDGM tekniği için MısDGm'nin hesaplanması Denklem 1 ile gösterilmektedir.

$$
\mathrm{M}_{\text {ISDGM }}=\frac{\mathrm{V}_{\mathrm{S}}}{\mathrm{V}_{\mathrm{T}}}
$$

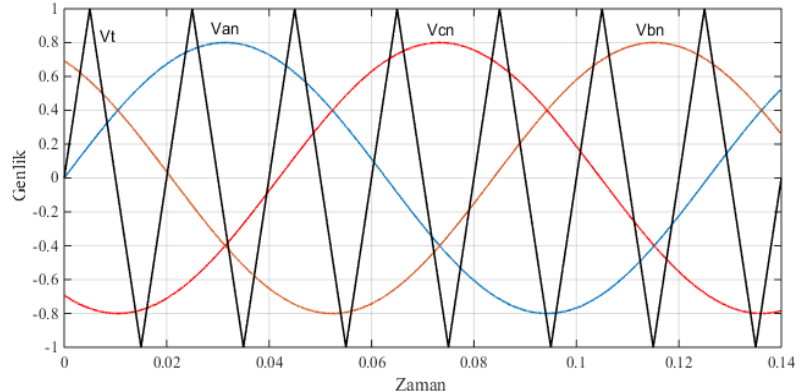

(a)
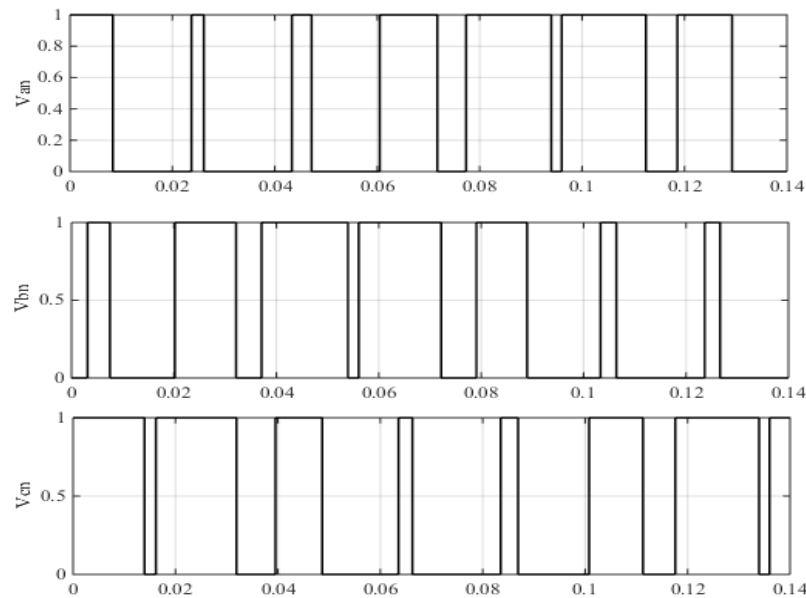

(b)

Şekil 3. Üç fazlı evirici (a) Giriş gerilim sinyalleri ve taşıyıcı sinyal, (b) Her üç faz için elde edilen sinyaller 


\section{Tam Köprü Evirici Devresinde HIDGM Yöntemi}

DGM tekniklerinde kazancı artırma yöntemlerinden biride SDGM yöntemi kullanılırken üçüncü harmoniğin eklenmesidir. Bu yöntemde, kontrol sinyali olarak kullanılan sinüs dalgasının üzerine üçüncü harmonik ilave edilerek taşıyıcı sinyal olan üçgen dalga ile karşılaştırılır. Üçüncü harmoniğin genliği referans gerilimin genliğinin 1/6'sı kadar artırılabilir. Bu durumda elde edilecek yeni kontrol sinyalin genliği Denklem 7 ile gösterilmektedir. Bu yöntem ile klasik SDGM yöntemine göre kazanç \%15 artırılabilmektedir [11,12]. Ancak, faz-nötr geriliminde yüksek dereceli 3. harmoniklerin bulunması dezavantajıdır.

\section{$\mathrm{V}^{*}=\mathrm{V} \cdot \operatorname{Sin} \omega \mathrm{t}+(1 / 6) \cdot \mathrm{V} \cdot \operatorname{Sin} \cdot 3 \omega \mathrm{t}$}

Şekil 4 (a), (b) ve (c)’de sırasıyla, sinüzoidal sinyale (1/6) oranında üçüncü harmoniğin enjekte edilmesi, kontrol sinyali ile taşıyıcı sinyalin bir arada karşılaştırılması en son olarak karşılaştırma sonucu elde edilen sinyal gösterilmektedir.

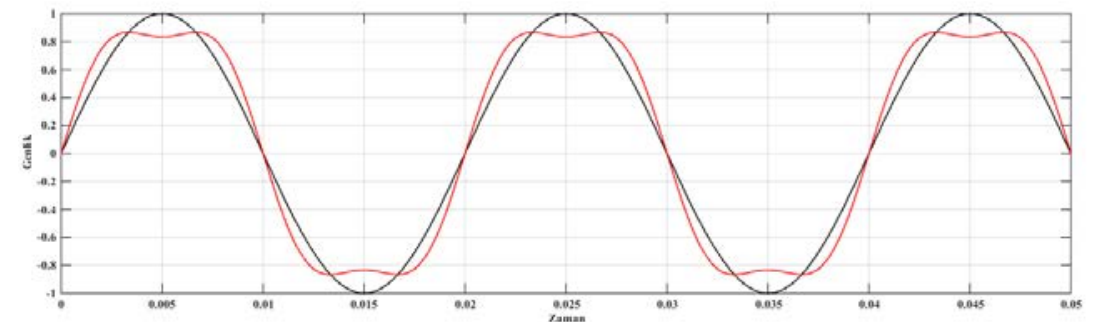

(a)

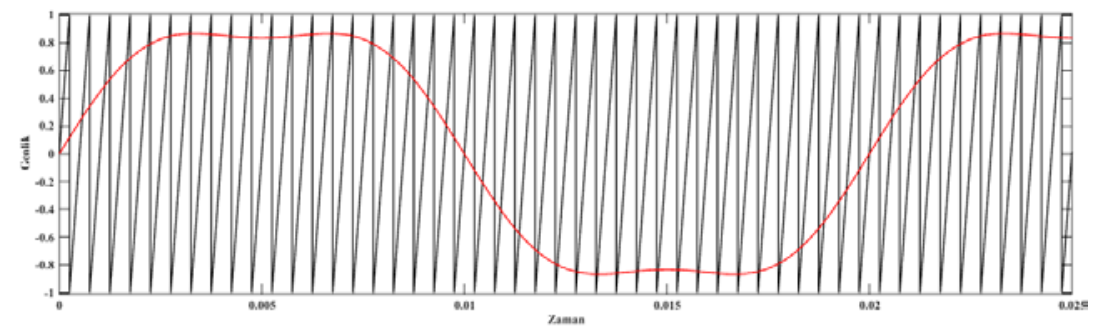

(b)

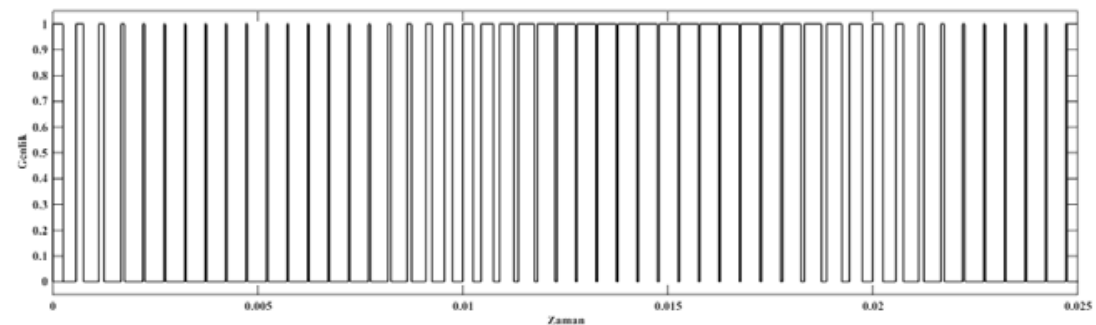

(c)

Şekil 4. (a)Sinüzoidal ve 3. Harmonik ilaveli sinyal, (b) Kontrol sinyalinin taşıyıcı sinyal ile karşılaştırılması, (c) HIDGM sinyali

\section{Tam Köprü Evirici Devresinde SHEDGM Yöntemi}

Bu çalışmada kullanılan SHEDGM ile akım veya gerilim dalga şeklini meydana getiren tüm kosinüs ve sinüs bileşenlerinin genlikleri ve frekansları belirlenerek Toplam Harmonik Dalgalanmanın (THD) azaltılması için gerekli eliminasyonlar gerçekleştirilmektedir. SHEDGM yöntemi programlanabilir DGM yöntemi olarak da adlandırılırken, güç kalitesinin artırılması, sürücü sistemleri, yüksek gerilim DA transfer (HVDC) sistemleri ve dağıtım sistemlerinde geniş bir uygulama alanlarına sahiptirler [8]. Seçili Harmonik Eliminasyon DGM (SHEDGM) yönteminde amaç; Fourier serisi açılımından yararlanarak akım ve gerilim dalga şekilleri içerisinde yer alan harmoniklerden bir yada bir kaçının genliğinin 
yok edilerek etkilerinin ortadan kaldırılmasıdır. Bu amaçla ya doğrusal olmayan denklem çözme yöntemlerinden biri (Newton metodu...), ya genetik algoritmalar (GA) gibi alternatif yöntemlerden biri yada her iki yöntemin bir arada kullanılmasıyla çıkış güç kalitesi artırılmaya çalışılmaktadır [6-10].

SHEDGM tekniğinde çıkış dalga şekli kare dalga simetrisine sahiptir. Bu durum Şekil 5 ile gösterilmekte ve $\alpha_{1}$, $\alpha_{2}, \alpha_{3}$ ve $\alpha_{4}$ açılarıyla belirlenen harmonikler yok edilir.

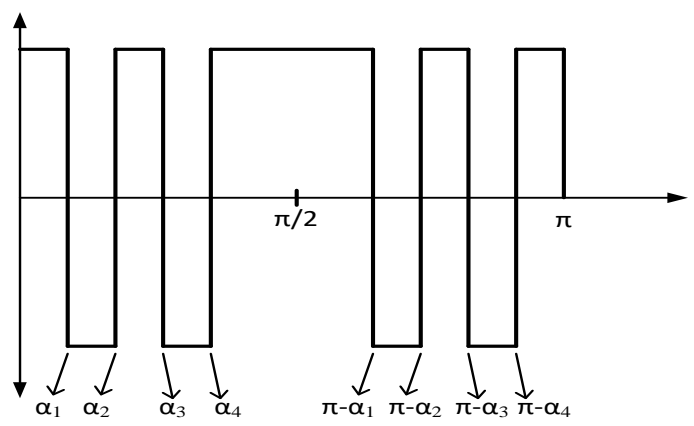

Şekil 5. Üç fazlı SHEDGM evirici dalga şekli

SHEDGM'de çıkış Alternatif Gerilimi (AG) tek yarım ve çeyrek dalga simetri özelliğine sahiptir. Bu nedenle de tüm çift harmonikler ve DA bileşeni sıfır olmaktadır $\left(n=2,4,6 \ldots v e V_{0 n}=0\right)$. Üç fazlı eviricinin çıkış faz-nötr gerilim dalga şekline ait Fourier serisinin genel bir açılımı Denklem 3, 4 ve 5 ile verilmektedir.

$\mathrm{V}(\mathrm{t})=\mathrm{a}_{0}+\sum_{\mathrm{n}=1}^{\infty}\left(\mathrm{a}_{\mathrm{n}} \operatorname{Cos} \omega \omega \mathrm{o}+\mathrm{b}_{\mathrm{n}} \operatorname{Sin} \omega \mathrm{t}\right)$

$\mathrm{a}_{\mathrm{n}}=\frac{1}{\pi} \int_{0}^{2 . \pi} \mathrm{V}(\mathrm{t}) \operatorname{Cos} \omega \cos \omega \mathrm{t}$

$\mathrm{b}_{\mathrm{n}}=\frac{1}{\pi} \int_{0}^{2 . \pi} \mathrm{V}(\mathrm{t}) \operatorname{Sin} \omega \mathrm{td} \omega \mathrm{t}$

Tek çeyrek kare dalga simetrisinde Fourier analizi uygulanarak n. tek harmonik genliği Denklem 4 ile verilen ifadeyle hesaplanabilirken çift harmoniklerin genlikleri sıfır olur $\left(a_{n}=0\right)$.

$$
\mathrm{V}(\mathrm{t})=\sum_{\mathrm{n}=1}^{\infty} \mathrm{b}_{\mathrm{n}} \operatorname{Sinn} \omega \mathrm{t}
$$

Denklem 5 ile verilen eşitlikte $b_{n}$ Denklem 7 ile hesaplanır.

$$
\left.\begin{array}{c}
\mathrm{b}_{\mathrm{n}}=\frac{4}{\pi}\left[\int_{0}^{\alpha_{1}} \frac{\mathrm{V}_{\mathrm{d}}}{2} \operatorname{Sinn} \omega \mathrm{td} \omega \mathrm{t}-\int_{\alpha_{1}}^{\alpha_{2}} \frac{\mathrm{V}_{\mathrm{d}}}{2} \operatorname{Sinn} \omega \mathrm{td} \omega \mathrm{t}+\ldots\right. \\
\left.\ldots-\ldots+\int_{\alpha \mathrm{n}}^{\pi / 2} \frac{\mathrm{V}_{\mathrm{d}}}{2} \operatorname{Sinn} \omega \mathrm{td} \omega \mathrm{t}\right]
\end{array}\right\}
$$

Herhangi bir açı değeri için genelleştirilmiş $b_{n}$ ise Denklem 8 ile verilmektedir.

$b_{n}=\frac{4 . V_{D}}{n \pi} \sum_{i=1}^{k}(-1)^{i+1} \operatorname{Cosn}_{i}$

Bu denklemde; k: çeyrek periyot anahtarlama açı sayısını, $\alpha_{1}<\alpha_{2}<\alpha_{3}<\alpha_{4}<\ldots<\pi / 2$ aralığındaki anahtarlama açı değerini ve n: tek harmonik derecesini göstermektedir. Örnek olarak, çeyrek periyotta dört anahtarlama durumu için yük geriliminin Fourier analizi yapılırsa Denklem 9 ile verilen eşitlik elde edilir.

$b_{n}=\frac{4 \cdot V_{D}}{n \pi} \cdot\left(\operatorname{Cos}\left(n \alpha_{i}\right)-\operatorname{Cos}\left(n \alpha_{2}\right)+\operatorname{Cos}\left(n \alpha_{3}\right)\right)$

Denklem 9 ile verilen ifadede $\alpha_{1}, \alpha_{2}$ ve $\alpha_{3}$ birbirinden bağımsız anahtarlama açılarını göstermektedir. Bu nedenle, bu anahtarlama açılarının her biri için doğrusal denklem yazılabilir. Genel olarak, en önemli düşük frekanslı harmonikler, yüksek frekanslı harmonik bileşenler ilave filtre devreleri kullanarak kolayca elenebilir. Harici harmonik sayısını sabit bir seviyede tutmak için, tüm anahtarlama açısı $\pi / 2$ 'den az olmalıdır. 


\section{Tam Köprü Evirici Devresinde UVDGM Yöntemi}

Uzay vektör DGM (UVDGM) yöntemi, dijital uygulamalarda kolay uygulanabilmeleri, düşük anahtarlama kayıplarına sahip olmaları ve çıkış faz-faz gerilimi için geniş lineer modülasyon çalışma alanları olmasından dolayı popüler DGM yöntemlerinden biridir. Klasik Sinüzoidal DGM yöntemine göre daha az harmonik içeriğe sahip olması ve güç kaynağının daha verimli kullanılması gibi üstünlüklerinin yanı sıra fazla hesaplama gerektirmesi ve karmaşıklığı dezavantajlara da sahiptir [5, 13].

Üç fazlı tam köprü evirici UVDGM yönteminde, Şekil 6 ile gösterilen motor gerilimini etkileyen toplam altı adet aktif gerilim vektörü $\left(\mathrm{V}_{1}, \mathrm{~V}_{2}, \mathrm{~V}_{3}, \mathrm{~V}_{4}, \mathrm{~V}_{5}, \mathrm{~V}_{6}\right)$ ve motor gerilimini etkilemeyen iki adet sıfır vektör $\left(\mathrm{V}_{0}, \mathrm{~V}_{7}\right)$ bulunmaktadır.

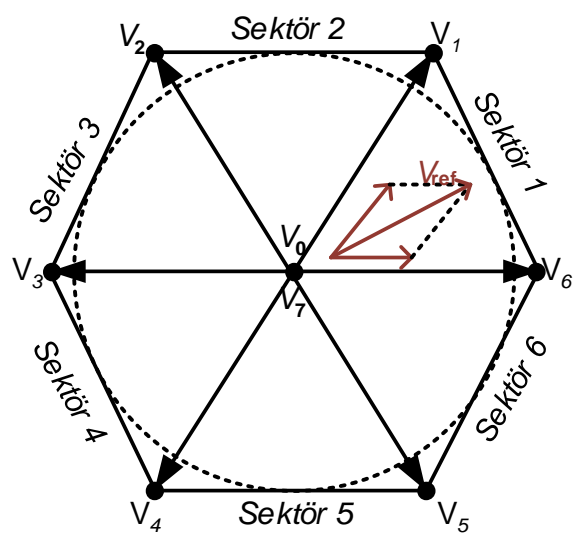

Şekil 6. Üç fazlı tam köprü eviriciye ait UVDGM diyagramı

Şekil 1 ile gösterilen evirici devresinde $\mathrm{S}_{\mathrm{a}^{+}}, \mathrm{S}_{\mathrm{b}+}$ ve $\mathrm{S}_{\mathrm{c}+}$ üst grup anahtarlar, $\mathrm{S}_{\mathrm{a}-}, \mathrm{S}_{\mathrm{b}-\text { ve }} \mathrm{S}_{\mathrm{c}-\text { alt grup anahtarlar olarak }}$ adlandırılacak olursa, anahtarların konum değişimlerinde üst grup anahtarlar göz önünde bulundurulur ve alt grup anahtarlar üst grup anahtarların “değili” olarak çalışır. Anahtarların iletimde olduğu durum “1”, kesimde olduğu durum “0” ile gösterilir. Bu durumda; a fazı, b fazı ve c fazına ait üst grup anahtarların sırasıyla konumları; $V_{1}=100, V_{2}=110$, $\mathrm{V}_{3}=010, \mathrm{~V}_{4}=011, \mathrm{~V}_{5}=001, \mathrm{~V}_{6}=101, \mathrm{~V}_{0}=000$ ve $\mathrm{V}_{7}=111$ olarak gösterilir.

UVDGM yönteminde, $\mathrm{V}_{\text {ref }}\left(\mathrm{V}^{*}\right)$; her bir anahtarlama sırasında kendisine en yakın (komşu) olan iki aktif gerilim vektörü ve sıfır gerilim vektörlerinin anahtarlamasıyla hesaplanır. Bu hesaplama yapılırken sırasyla;

$>\mathrm{V}_{\mathrm{d}}, \mathrm{V}_{\mathrm{q}}, \alpha$ ve $\mathrm{V}_{\text {ref }}$ değerleri hesaplanır,

$>\quad \alpha$ 'nın değerine göre $V_{\text {ref' }}$ in yer aldığı sektör belirlenir,

> Her bir anahtarlama durumu için $\mathrm{T}_{1}, \mathrm{~T}_{2}$ ve $\mathrm{T}_{0}$ süreleri hesaplanır,

$>$ Anahtarlama yönü ve sırası tespit edilerek gerilim üretilir.

Anahtarlama sıralaması belirlenirken de durum değişimleri sırasında sadece bir anahtarın konum değiştirmesi kayıpların ve harmonik bozulmanın az olmasına sebep olacaktır. Bu durum bir örnek ile özetlenecek olursa; Sektör 3'de $T_{s} / 2$ süresince sırasıyla $V_{0}, V_{3}, V_{5}$ ve $V_{7}$ ardından $T_{s} / 2$ süresince sırasıyla $V_{7}, V_{5}, V_{3}$ ve $V_{0}$ gerilim vektörleri kullanılarak $\mathrm{T}_{\mathrm{s}}$ süresi tamamlanır.

\section{Benzetim Modeli ve Sonuçları}


Şekil 7'de, sırasıyla SDGM, HIDGM, SHEDGM ve UVDGM yöntemleri kullanılan üç fazlı iki seviyeli evirici devresinin MATLAB benzetim modeli gösterilmektedir. Bu modelde; "DGM Yöntemi” bloğu içerisinde sırasıyla her bir çalışma durumu için SDGM, HİDGM, SHEDGM ve UVDGM yöntemleri modellenmiştir. "3 Fazlı Evirici Devresi” bloğunda her faz için 2 anahtar kullanılarak toplamda 6 anahtar ve harici bir DA kaynağına bağlı 3-fazlı evirici devresi Şekil 1'de gösterilen devre yapısına uygun olarak Simulink ortamında modellenmiştir. Yük bloğunda ise her biri $\mathrm{R}=100 \Omega$ ve $\mathrm{L}=0.1 \mathrm{H}$ olan üç fazlı bir yük kullanılmaktadır. Tüm çalışma şartlarına modülasyon indeks değeri 0.8 ve anahtarlama frekansı $1 \mathrm{kHz}$ olarak belirlenmiştir.

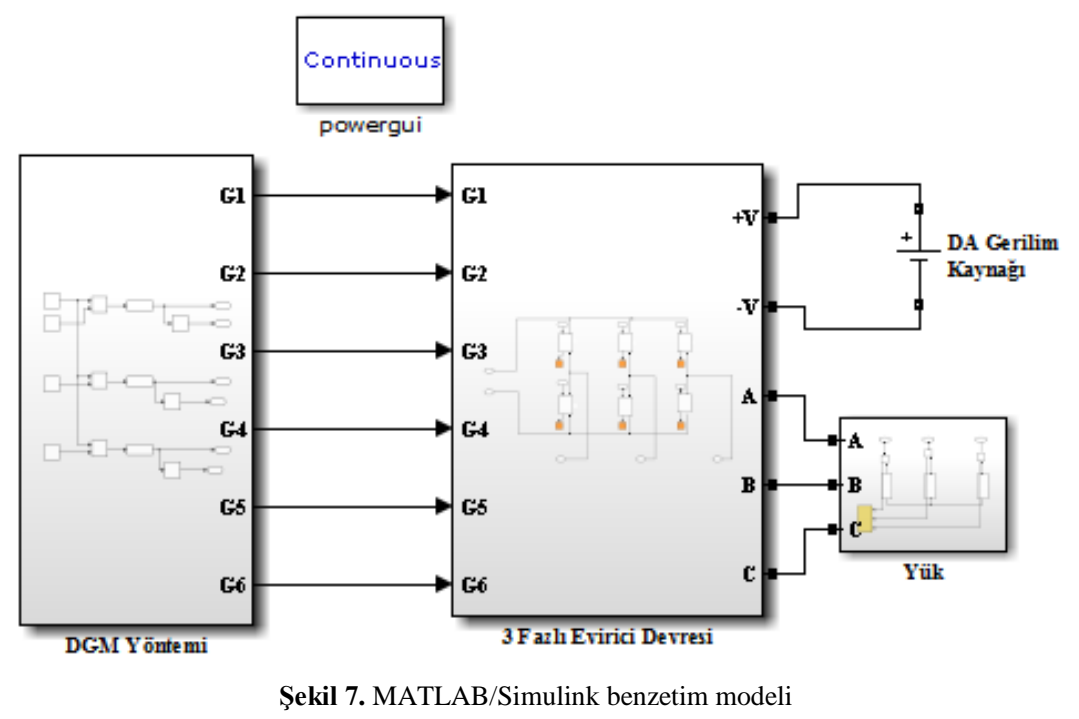

\subsection{SDGM Yöntemi ve Dalga Şekilleri}

Şekil 8 (a) ile SDGM yöntemi kullanılan üç fazlı iki seviyeli evirici devresinin akım dalga şekli ve gerilim dalga şekli bir arada gösterilmektedir. Ayrıca akım ve gerilim dalga şekillerinin Toplam Harmonik Analizi Şekil 8 (b) ve (c)'de sirasıyla verilmektedir.

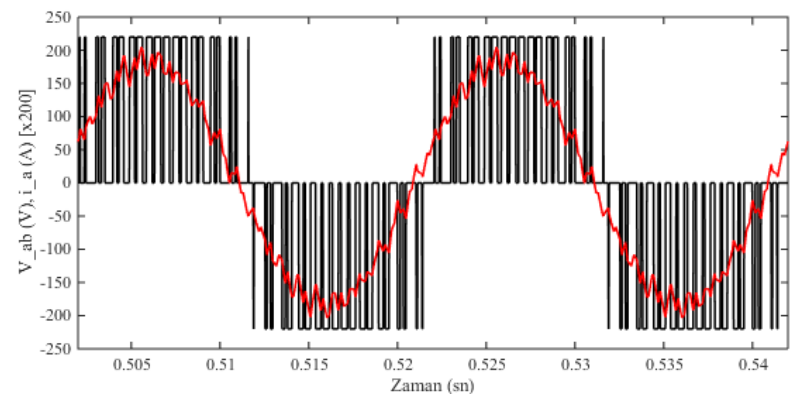

(a)

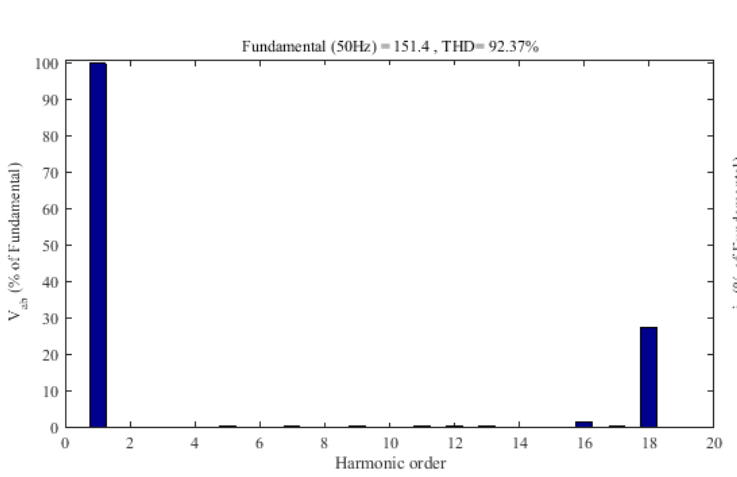

(b)

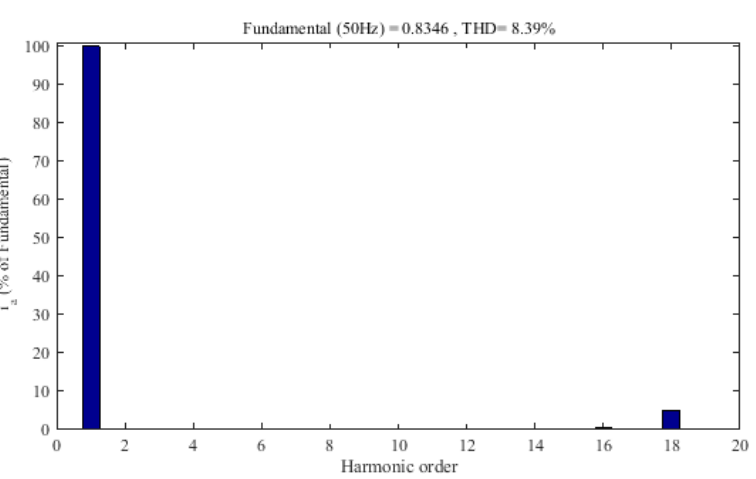

(c) 
Nevşehir Bilim ve Teknoloji Dergisi (2018), 7(1) 100-110

Şekil 8. $\mathrm{m}=0.8$ ve $\mathrm{f}=50 \mathrm{~Hz}$ için için SDGM yöntemi kullanılan 3 fazlı evirici (a) çıkış hat gerilimi (a) çıkış hat gerilimi ( $\left.\mathrm{V}_{\mathrm{ab}}\right)$ ve hat akımı ( $\left.\mathrm{i}_{\mathrm{a}}\right)$, (b) gerilimin harmonik spektrumu, (c) akımın harmonik spektrumu

\subsection{HiDGM Yöntemi ve Dalga Şekilleri}

Şekil 9 (a), (b) ve (c)'de sırasıyla HIDGM yöntemi kullanılan üç fazlı iki seviyeli evirici devresinin akım dalga şekli ve gerilim dalga şekli, gerilim THD ve akım THD bir arada verilmektedir.

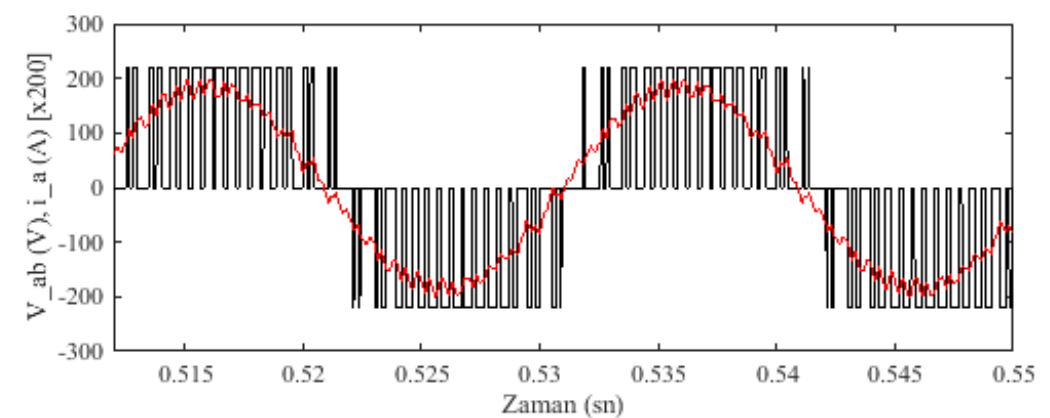

(a)

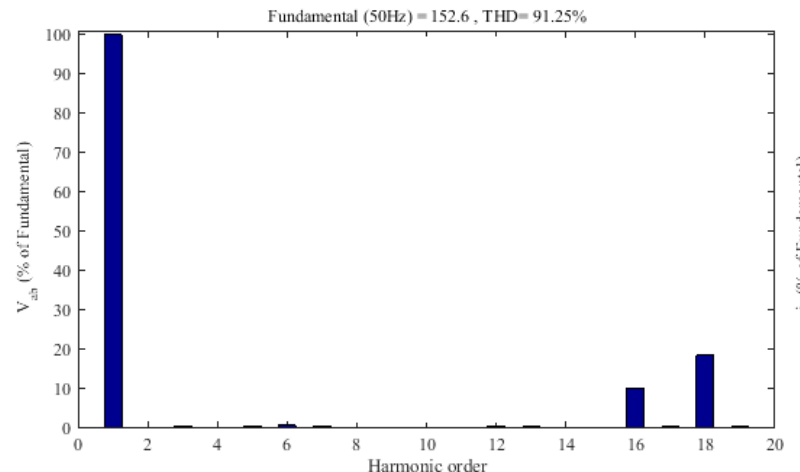

(b)

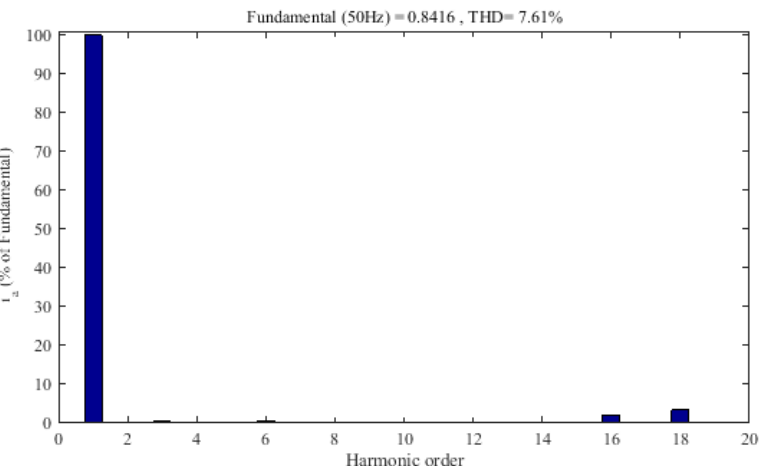

(c)

Şekil 9. $\mathrm{m}=0.8$ ve $\mathrm{f}=50 \mathrm{~Hz}$ için HIDGM yöntemi kullanılan 3 fazlı evirici (a) çıkış hat gerilimi $\left(\mathrm{V}_{\mathrm{ab}}\right)$ ve hat akımı ( $\mathrm{i}_{\mathrm{a}}$ ), (b) gerilimin harmonik spektrumu, (c) akımın harmonik spektrumu

\subsection{SHEDGM Yöntemi ve Dalga Şekilleri}

Şekil 10 (a) ile SHEDGM yöntemi kullanılan üç fazlı iki seviyeli evirici devresinin akım dalga şekli ve gerilim dalga şekli bir arada gösterilmektedir. Gerilm THD ve akım THD ise Şekil 10 (b) ve (c) ile gösterilmektedir.

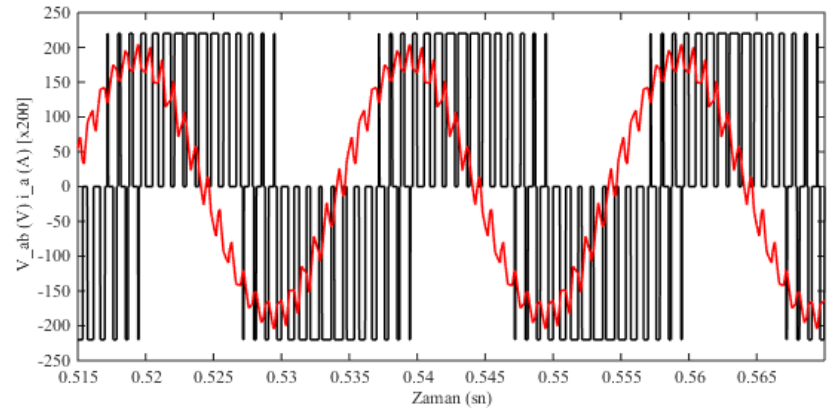

(a) 


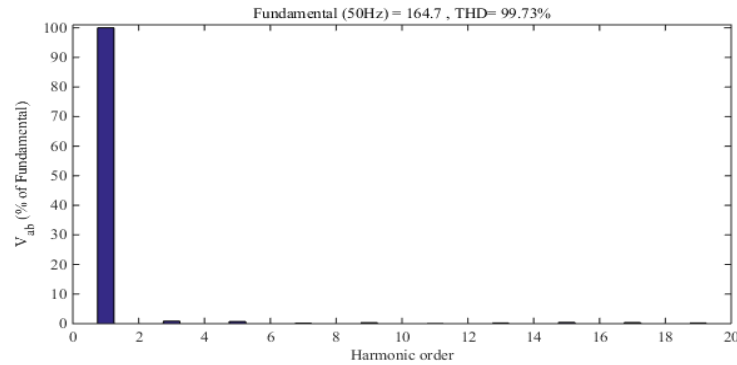

(b)

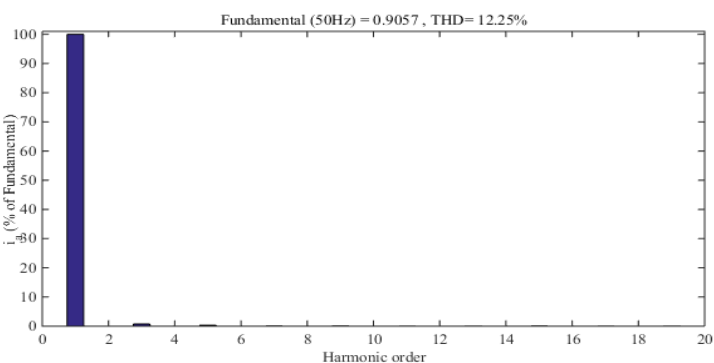

(c)

Şekil 10. $\mathrm{m}=0.8$ ve $\mathrm{f}=50 \mathrm{~Hz}$ için için SHEDGM yöntemi kullanılan 3 fazlı evirici (a) çıkış hat gerilimi (a) çıkış hat gerilimi $\left(\mathrm{V}_{\mathrm{ab}}\right)$ ve hat akımı (ia $)$, (b) gerilimin harmonik spektrumu, (c) akımın harmonik spektrumu

\subsection{UVDGM Yöntemi ve Dalga Şekilleri}

Şekil 11 (a) ile SVDGM yöntemi kullanılan üç fazlı iki seviyeli evirici devresinin akım dalga şekli ve gerilim dalga şekli bir arada gösterilmektedir. Ayrıca akım ve gerilim dalga şekillerinin Toplam Harmonik Analizi Şekil 8 (b) ve (c)'de sirasiyla verilmektedir.

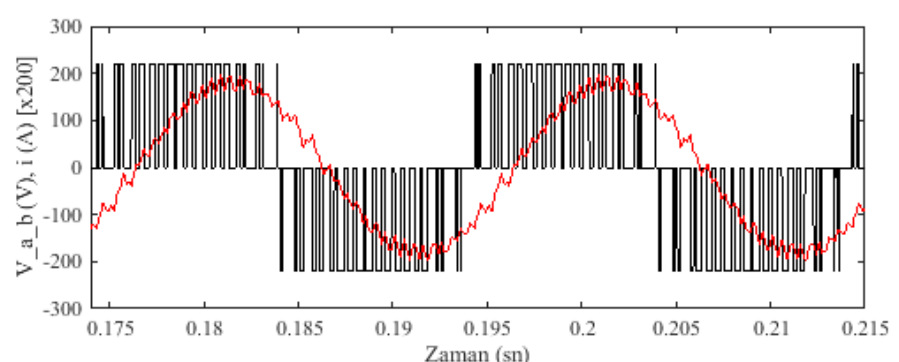

(a)

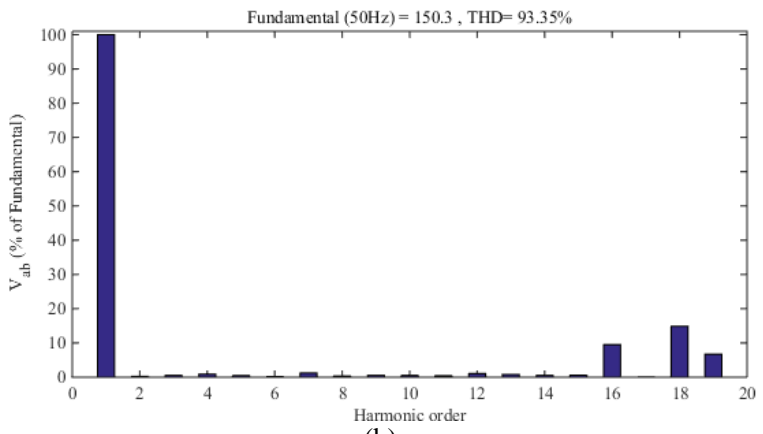

(b)

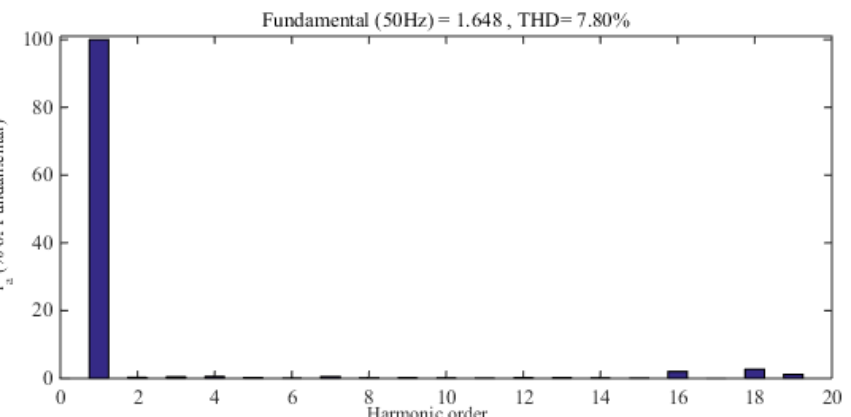

(c)

Şekil 11. $\mathrm{m}=0.8$ ve $\mathrm{f}=50 \mathrm{~Hz}$ için için UVDGM yöntemi kullanılan 3 fazlı evirici (a) çıkış hat gerilimi (a) çıkış hat gerilimi $\left(\mathrm{V}_{\mathrm{ab}}\right)$ ve hat akımı (i $\left.\mathrm{i}_{\mathrm{a}}\right)$, (b) gerilimin harmonik spektrumu, (c) akımın harmonik spektrumu

7

\section{Sonuçlar}

Bu çalışmada, üç fazlı iki seviyeli gerilim kaynaklı bir evirici devresinde Sinüzoidal Darbe Genişlik Modülasyon Tekniği, Üçüncü harmonik İlaveli Darbe Genişlik Modülasyon Tekniği, Seçili Harmonik Eliminasyon Darbe Genişlik Modülasyon Tekniği ve Uzay Vektör Darbe Genişlik Modülasyon Tekniği kullanılarak evirici anahtarlarının iletim/kesim durumlarına göre çıkış gerilimi ve akımı incelenmiştir. Çalışma sırasında modülasyon indeks değeri 0.8 ve çıkış frekans değeri $50 \mathrm{~Hz}$, yük değeri ise $\mathrm{R}=100 \Omega, \mathrm{L}=0.1 \mathrm{H}$ olarak sabit kabul edilmiştir.

Gerek asenkron motorlar gerek diğer sürme devrelerinde kullanılan anahtarlar vasıtasıyla harmonik içerikler artmaktadır. Ancak günümüzde yarı iletken teknolojisindeki gelişmelerle beraber anahtarlar daha hızlı ve anahtarlama kayıpları daha az olmaktadır. Ayrıca kullanım alanlarına göre değişik DGM teknikleri ile kazancın artırılması da sağlanmaktadır. SDGM, HIDGM, SHEDGM ve UVDGM tekniklerinin her birinin kullanım alanına göre avantajları vardir.

SDGM yöntemi birçok açıdan modellemesi, sayısal olarak üretilebilir olması ve fazla matematiksel hesaplamaya ihtiyaç duymaması sebepleriyle ilk tercih edilecek yöntem olmasına karşın maksimum veriminin giriş geriliminin \%86 ile sınırlandırılmasından dolayı yeni yöntemlerin geliştirilmesi üzerinde durulmuştur. HIDGM tekniği SDGM tekniğine 
yaklaşık \%15 daha verimli çalışırken UVDGM tekniğine göre daha az hesaplama gerektirir. Ancak aşırı modülasyon bölgesinde ve kare dalga çalışmada SDGM yöntemi ile benzer sorunlarla karşılaşılır. SHEDGM yöntemin de ise gerekli işlemlerin yapılabilmesi isin başlangıç değerlerinin iyi tahmin edilmesi gerekmektedir. Son yıllarda SHEDGM için Bulanık mantık, Genetik Algoritma ve benzeri yöntemleri kullanılarak bu sorunun giderilmesi üzerinde çalışmalar artmıştır. UVDGM yönteminde ise referans gerilimin vektör eksenlerin üzerinde dönüşü ile gerilim üretilmektedir. İki seviyeli evirici devresinde vektörün hareket ettiği bölgeyi tespit etmek fazla matematiksel işlem gerektirmese de daha yüksek seviyeler işlem yükü artmaktadır.

\section{Kaynaklar}

[1] Malik S., Kluge D., “ACS1000 World's First Standard AC Drive for Medium-Voltage Applications”, ABB Review, No. 2,4-11, 1998.

[2] Rodriguez, J., Lai, J.S., and Peng, F.Z. "Multilevel Inverter: A Survey of Topologies, Controls and Applications", IEEE Transactions on Industrial Electronics, 49(4) , 724-738, 2002.

[3] Patel, H.S., Hoft, R.G., "Generalized Techniques of Harmonic Elimination and Voltage Control in Thyristor Inverters: Part IHarmonic Elimination”, IEEE Trans. on Industry Applications, IA-9(3), 310-318, 1973.

[4] Kouro, S., Malinowski, M., Gopakumar, K., Pou, J., Franquelo, L., Wu, B., Rodriguez, J., P'erez, M.A. ve Leon, J., "Recent Advances and Industrial Applications of Multilevel Converters,” IEEE Transactions on Industrial Electronics, 57(8), 2553-2580, 2010.

[5] Kocalmis Bilhan, A., Sunter, S., "Simulation of a Space Vector PWM Controller For a Three-Level Voltage-Fed Inverter Motor Drive”, IECON2006, 7-10 Kasim, 1915-1920, Paris, 2006.

[6] Deniz, E., “İki Seviyeli SHEPWM İnverter için Genetik Algoritma Kullanılarak Anahtarlama Açılarının Belirlenmesi”, Fırat Üniversitesi Müh. Bil. Dergisi, 27(10), 35-42, 2015.

[7] Abdelhamid, T.H. ve El-Naggar, K.M. ”Optimal PWM Control of a New Generalized Family of Multilevel Inverters”, Taylor \& Francis, Electric Power Components and Systems, 36(1), 73-92, 2008.

[8] Li, L., Czarkowski, D., Liu, Y.,ve Pillay, P,”Multilevel Selective Harmonic Elimination PWM in Series Connected Voltage Inverters", Transactions on Industrial Electronics, 36(1), 160-170, 2000.

[9] Aguilera, R., Lezana, P., Konstantinou, G., Acuna, P., Wu, B., Bernet, S., Agelidis, V.G., “Closed-Loop SHE-PWM Technique for Power Converters Through Model Predictive Control”, IECON2015, 5261-5266, 9-12 Kasım, 2015.

[10] Rodriguez, J., Kazmierkowski, M.P., Espinoza, J., Zanchetta, P., Abu- Rub, H., Young, H.A. ve. Rojas, C.A “State of the Art of Finite Control Set Model Predictive Control in Power Electronics,” IEEE Transactions on Industrial Informatics, 9(2), 1003-1016, May1s, 2013.

[11] Massaoud, A.M., Finner, S.J., Williams, B.W., “Control Techniques for Multilevel Voltage Source Inverters”, 34th Annual IEEE Power Electronics Specialists Conf., 171-176, Mexico 2003.

[12] Yumurtac1, M., Varbak Neşe, S., Üstün, S.V., Oğuz, Y., “THIPWM ile Kontrol Edilen 3 Fazlı Gerilim Beslemeli İnverterin Matlab/Simulink Modellenmesi ve DSP Kontrolör ile Uygulamasının Gerçekleştirilmesi”, IATS '09,13-15 Mayıs, Karabük, 2009.

[13] Celenovic, N., “Space Vector Modulation and Control of Multilevel Converter”, Doktora tezi, Faculty of the Virginia Polytechnic Institute and State University, September, 2000. 


\section{Extended Abstract}

\section{Introduction}

In recent years, the rapid increase in electricity consumption and the limited availability of fossil energy sources have prompted researchers to explore alternative sources of energy and how to use existing energy more efficiently. For this purpose, renewable energy sources such as solar batteries, wind generators, wind turbines, fuel cells and etc. have been started to be used more and more widely. However, Direct Current (DC), which is produced by using renewable energy sources or by using rectifiers, needs to be converted to Alternative Current (AC) in the industry and many other areas. For this purpose, power electronic circuits, called inverters, are used. Basically, inverters are used to produce a desired alternative voltage from a direct voltage source. Nowadays inverters are used in approximately $85 \%$ of the drive systems applications such as pumps, conveyor belt mists, fans and etc [1-4]. When the entire system is evaluated inverters reduces energy loss considerably.

The most important feature expected from inverters during this conversion is that the desired voltage and operating frequency are achieved at the output and the resulting voltage/current distortion is at a low level. The inverter input voltage can be obtained from sources such as solar panels, batteries, wind turbines, as well as from the rectifier circuit. Fig.1 shows a schematic drawing of a three-phase inverter circuit.

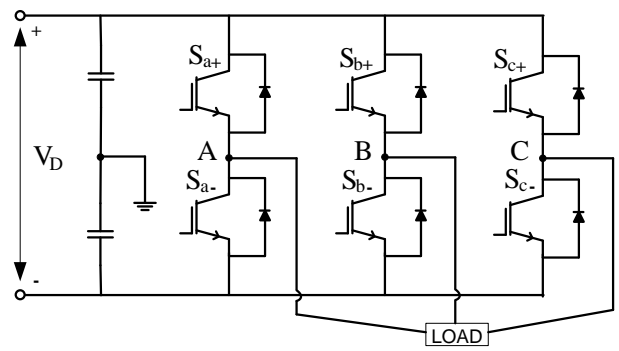

Fig.1. Three-phase inverter circuit

Various techniques called Pulse Width Modulation (PWM) Techniques have been developed such as Sinusoidal Pulse Width Modulation Technique (SPWM), Selective Harmonic Elimination Pulse Width Modulation Technique, Harmonic Injection Pulse Width Modulation Technique (HIPWM), Space Vector Pulse Width Modulation Technique (SVPWM), Sigma-Delta Pulse Width Modulation Technique (SVPWM) and etc. to adjust the amplitude and frequency of the output voltage in the inverter circuit shown in Figure 1 [5-13]. With each developed pwm techniques, It is aimed to reduce the switching losses, to extend the linear modulation range and to reduce the harmonics in the voltage and current waveforms, especially at the inverter output, and to control the amplitude frequency of the fundamental harmonic component [6].

\section{Method}

In this work, Sinusoidal Pulse Width Modulation Technique (SPWM), Selective Harmonic Elimination Pulse Width Modulation Technique, Harmonic Injection Pulse Width Modulation Technique (HIPWM) and Space Vector Pulse Width Modulation Technique (SVPWM) for a three phase voltage-fed inverter has been modelled and simulated respectively. Simulation results have been given for $\mathrm{R}=100 \Omega$ and $\mathrm{L}=0.1 \mathrm{H}$ load. Switching frequency of $1 \mathrm{kHz}$ and the modulation index of 0.8 were used in the model. DC link voltage of the inverter was taken as $200 \mathrm{~V}$.

\section{Results and Discussion}

Harmonic contents are increasing due to the use of asynchronous motors and switches in driving circuits. Today, the improvements in semiconductor technology are faster and the switching losses are less. It is also possible to increase the stability with different PWM techniques according to application areas. Each of modulation technique (SPWM, HIPWM, SHEPWM and UVPWM techniques) has advantages over the field of use.

Although the SPWM method is the first choice method due to the fact that it can be digitally generated and does not require over mathematical computation, the maximum output is limited to $86 \%$ of the input voltage. Therefore, the new methods are developed such as HIPWM, SHEPWM and etc. 